\title{
Concentrations of Plasma Immunoglobulins in the Dog as Determined by Laser Nephelometry. Comparison with Radial Immunodiffusion and Enzyme-Linked Immunosorbent Assay
}

\author{
Pedro J. Ginel, Juan M. Margarito, Rosario Lucena and José M. Molleda \\ Departmento de Patología Clínica Veterinaria, Facultad de Veterinaria, Medina Azahara s/n, Córdoba, España
}

Summary: Concentrations of immunoglobulins were determined by laser nephelometry and, for comparison, by single radial immunodiffusion assay and a sandwich enzyme-linked immunosorbent assay (ELISA) in plasma samples of normal dogs.

Correlation between methods was high, although the mean IgM concentrations determined by laser nephelometry were significantly higher than those determined by the single radial immunodiffusion assay or ELISA. In contrast, the mean IgA concentrations were significantly lower than those determined by ELISA. Laser nephelometry was the most reproducible method. The between-assay coefficient of variation was $4.12 \%$ for IgG, $6.98 \%$ for IgM and $6.35 \%$ for IgA. Laser nephelometry and ELISA showed similar accuracies, and both were more accurate than single radial immunodiffusion. Finally, laser nephelometry was much more sensitive than single radial immunodiffusion, but less sensitive than the ELISA method.

In conclusion, results of the three methods were in general comparable. The higher range of linearity, precision and accuracy of laser nephelometry, and the availability of automated nephelometers make this the method of choice for quantifying canine plasma immunoglobulins.

\section{Introduction}

The measurement of serum or plasma immunoglobulin concentrations is one of the commonest tests carried out in human and veterinary immunology. All the methods available for quantifying immunoglobulins can be classified into two major categories (1): those involving measurement of the physical properties of the immune complexes formed between the antigen and the antiserum, i. e. turbidimetry, light scattering (nephelometry) and precipitation in gel (radial immunodiffusion), and those in which the amount of labelled antibody bound to the antigens is measured as in the enzyme-linked immunosorbent assays (ELISA).

For many years the single radial immunodiffusion assay has been the method of choice, although for analysis of human samples it has been replaced by ELISA and especially by laser nephelometry. Nephelometry has the advantage of being a highly automated method which produces faster results and can handle a large number of samples. On the other hand, the cost of the nephelometer has limited its use in veterinary practice and to the author's knowledge there are few reports of its use for determining plasma immunoglobulin concentrations in the dog. Thus, the sandwich ELISA is the preferred alternative to single radial immunodiffusion assay for quantifying individual immunoglobulins, but the ELISAs are more time consuming than nephelometry.

In the present study, laser nephelometry was used to measure normal plasma concentrations of canine IgG, IgM and IgA. Test results were compared with those obtained by single radial immunodiffusion assay and by an ELISA method.

\section{Materials and Methods}

Forty-four clinically healthy, household dogs were used. The study population comprised 15 male and 29 female dogs of various breeds, with ages ranging from 6 months to 8 years, and these were used to establish the normal range of immunoglobulin values. Blood was collected from all dogs by venepuncture into heparincontaining vials. Immediately after collection, the blood was centrifuged $(3000 \mathrm{~g}, 15 \mathrm{~min}$, room temperature) and the resulting plasma samples were stored at $-20^{\circ} \mathrm{C}$ in $0.5 \mathrm{ml}$ aliquots and analysed.

Antisera used for laser nephelometry, single radial immunodiffusion and ELISA techniques included the purified IgG fractions from rabbit anti-canine IgA, IgG or IgM antiserum; for the analytical recovery experiments purified dog immunoglobulins were used. Antisera and purified immunoglobulins were a kind gift from Dr. W. E. Bernadina, Utrecht. Preparation of the immunoglobulins and antisera has been described previously (2). Briefly, IgA was isolated from a dog with multiple myeloma by chromatography over AcA22 (LKB, Stockholm). Immunoglobulin $G$ and $M$ were purified from ascites fluid and serum by delipidation, ammonium sulphate precipitation, dialysis against distilled water and gel filtration 
on AcA22. The supernatant was chromatographed on a protein ASepharose-EL-4B column (Pharmacia, Sweden). Antisera were raised in rabbits by inoculation of the antigens mixed with Freund's adjuvant. The antisera were made specific by solid absorption with antigen covalently bound to $\mathrm{CNBr}$ activated Sepharose-C1-4B (Pharmacia, Sweden).

For use as second antibody in the ELISA, the antisera were each biotinylated using $D$-biotinyl- $\gamma$-aminocaproic acid-N-hydroxysuccinimide ester (Biotin-X-NHS, Boehringer Mannheim), essentially according to the manufacturer's instructions. For quantitation of canine IgA, IgG or IgM commercially available reference standards (Miles Laboratories, Elkhart, Ind.) were used at optimal dilutions in each assay.

\section{Laser nephelometry procedure}

The method employed was basically that described by Goodswaard et al. (1980) (3). A nephelometer (Hyland Division, Travenol Lab. Inc., Costa Mesa) which incorporates a $\mathrm{He} / \mathrm{Ne}$ laser as the light source and an optical system detecting forward light scatter was used. Previously determined optimal dilutions of the antisera were $1: 30$ for IgG, $1: 25$ for IgM and $1: 10$ for IgA. The working antisera were filtered through a $0.4 \mu \mathrm{m}$ polycarbonate membrane (Nucleopore Corp., Pleasanton) to remove particulate matter, and the saline blank was filtered by the same method. For each assay system, $1 \mathrm{ml}$ of the working antiserum was added to a series of 10 $\times 75 \mathrm{~mm}$ disposable cuvettes and labelled as a test sample. One $\mathrm{ml}$ of the saline was added to a separate series of cuvettes for each reference and test sample blank. Saline blank and antiserum blank were prepared by pipetting $1 \mathrm{ml}$ of saline and antiserum respectively into the cuvettes. The reference standard was used in at least five different dilutions in saline. Optimal dilutions of the samples were $1: 150$ for IgG, and 1:25 for IgM and IgA. Dilutions of plasma samples were made in polyethylenglycol buffer which decreases the solubility of high-molecular-mass antibody/antigen complexes, allowing rapid measurement and an extended range of linearity. Dilutions of standards and samples were pipetted $(25 \mu \mathrm{l})$ into the sample blank and test sample cuvettes. Incubation and measurement of the percentage of relative light scatter were carried out as described by Deaton et al. (1976) (4). The readings of relative light scatter are made by quasi-equilibrium analysis, i.e. by measuring light scattered by immune complexes in reaction mixtures immediately after addition of the antiserum (blank) and then again after 1 hour incubation with the antisera. The final readout is proportional to the amount of antigen/antibody complexes present in the sample test cuvette.

\section{Single radial immunodiffusion procedure}

Basically the method of Mancini et al. (1965) (5) was followed. Antisera were incorporated into 1\% agarose (Seakam FMC Bioproducts, Rockland) and poured on $10 \times 15 \mathrm{~cm}$ glass plates at a rate of $2 \mu \mathrm{l}$ of antiserum per $\mathrm{cm}^{2}$. Wells of $4 \mathrm{~mm}$ diameter were punched out and $10 \mu \mathrm{l}$ from each sample and four dilutions of the reference standards were loaded at two positions on each plate. After incubation ( 72 hours), the plates were stained and ring diameters were measured using a graded optical micrometer. Immunoglobulin values were determined from a reference curve of ring diameter (in millimeters) versus the corresponding standard.

\section{ELISA procedure}

The assay was described recently (6). Briefly, microtitre Nunc plates (Maxisorb Nunc, Denmark) were coated with canine Ig-capturing antibody in $0.1 \mathrm{~mol} / 1$ sodium carbonate buffer, $\mathrm{pH} 9.6$ and blocked with $5 \mathrm{~g} / 1$ bovine serum albumin (Sigma Chemical, St Louis) in phosphate-buffered saline ( $0.01 \mathrm{~mol} / 1, \mathrm{pH} 7.2)$. Standards and plasma samples were added to duplicate wells and the biotinlabelled antiserum to canine IgG, IgM, or IgA was added to the appropriate wells. Bound biotinylated antibodies were detected as follows. Freshly prepared streptavidin-peroxidase conjugate (Sigma Chemical, St Louis) was diluted 4000 -fold, $100 \mu$ aliquots per well, and incubated for $1 \mathrm{~h}$ at $37^{\circ} \mathrm{C}$. The peroxidase reaction was determined after a $15 \mathrm{~min}$ incubation at room temperature with 2,2'-azino-di-3-ethyl-benzothiazoline sulphonic acid (Sigma Chemical, St Louis) and 35\% hydrogen peroxide (ABTS $+\mathrm{H}_{2} \mathrm{O}_{2}$; $0.5 \mathrm{mg}+1 \mu \mathrm{l} \mathrm{per} \mathrm{ml} \mathrm{of} 0.1 \mathrm{~mol} / \mathrm{l}$ citrate buffer, $\mathrm{pH}$ 5). The absorbance of the peroxidase reaction was measured at $405 \mathrm{~nm}$ using a microtitre plate reader (Titertek multiscan, Flow Laboratories, Ayrshire, UK).

\section{Assay evaluation}

Linearity of the test results on standards and test plasma samples was evaluated by least-squares regression analysis (7). Recovery of each immunoglobulin class was assessed by assaying canine sera with known contents of the immunoglobulin classes studied versus the same sera following addition of a known amount of each immunoglobulin class. Assay-to-assay variability was estimated by performance of duplicate determinations on the same standards over 10 separate assays. Sensitivity (i.e. the positive boundary) of the assay was calculated from the standard curves, being the immunoglobulin concentration value giving a relative light scatter or $405 \mathrm{~nm}$ absorbance value equal to that of the blank's mean +2.6 standard deviations (8). Normality of immunoglobulin concentrations was assessed by the Kolmogorov-Smirnov test. Correlations between test results were evaluated by use of the Spearman rank correlation analysis. Mean immunoglobulin concentrations determined by each test were compared using one-way non-parametric analysis of variance (7).

\section{Results}

A standard curve for laser nephelometry was constructed by plotting, on a linear scale, the relative light scatter of the reference standard dilutions versus their concentrations for all canine immunoglobulin classes tested (fig. 1 a to $\mathrm{c}$ ). The test response curve for increasing dilutions of each immunoglobulin class displayed a nearly linear portion (fig. 2). When responses to the reference plasma were assessed on 10 separate occasions, the resulting standard curves did not vary significantly. The plasma immunoglobulin concentration was calculated from a least-squares regression line of the standard curve. The between assay coefficient of variation (CV) was lower for IgG than for IgM and IgA (tab. 1). The technique showed a low detection limit and the average analytical recovery was high for all the immunoglobulins (tab. 1).

To obtain the single radial immunodiffusion values, a standard curve was constructed by plotting, on a linear scale, the diameter of the reference standard dilutions versus their concentration for all canine immunoglobulin classes tested. The between assay variation was similar for IgG and IgA and lower for IgM. As expected, the method was less sensitive than laser nephelometry, whereas the average analytical recovery was similar for both methods (tab. 1).

A standard curve for the ELISA assay was constructed using the logit-log transformation of the $405 \mathrm{~nm}$ absorbance values of the reference plasma dilutions steps. The plasma immunoglobulin content was determined from a least-squares regression line of the logit-log curve. Assay-to-assay variation was low for IgM and acceptable for IgG and IgA. Average analytical recovery ranged 
from $95.4 \%$ for IgA to $98.1 \%$ for IgM. Detection limits for the different immunoglobulins studied demonstrated the high sensitivity of the method (tab. 1).

\section{Plasma immunoglobulin concentrations}

Laser nephelometry, single radial immunodiffusion assay and ELISA were used to measure the concentrations of IgG, IgM and IgA in plasma samples from the 44 dogs. The results are shown in table 2 . The concentrations ob-
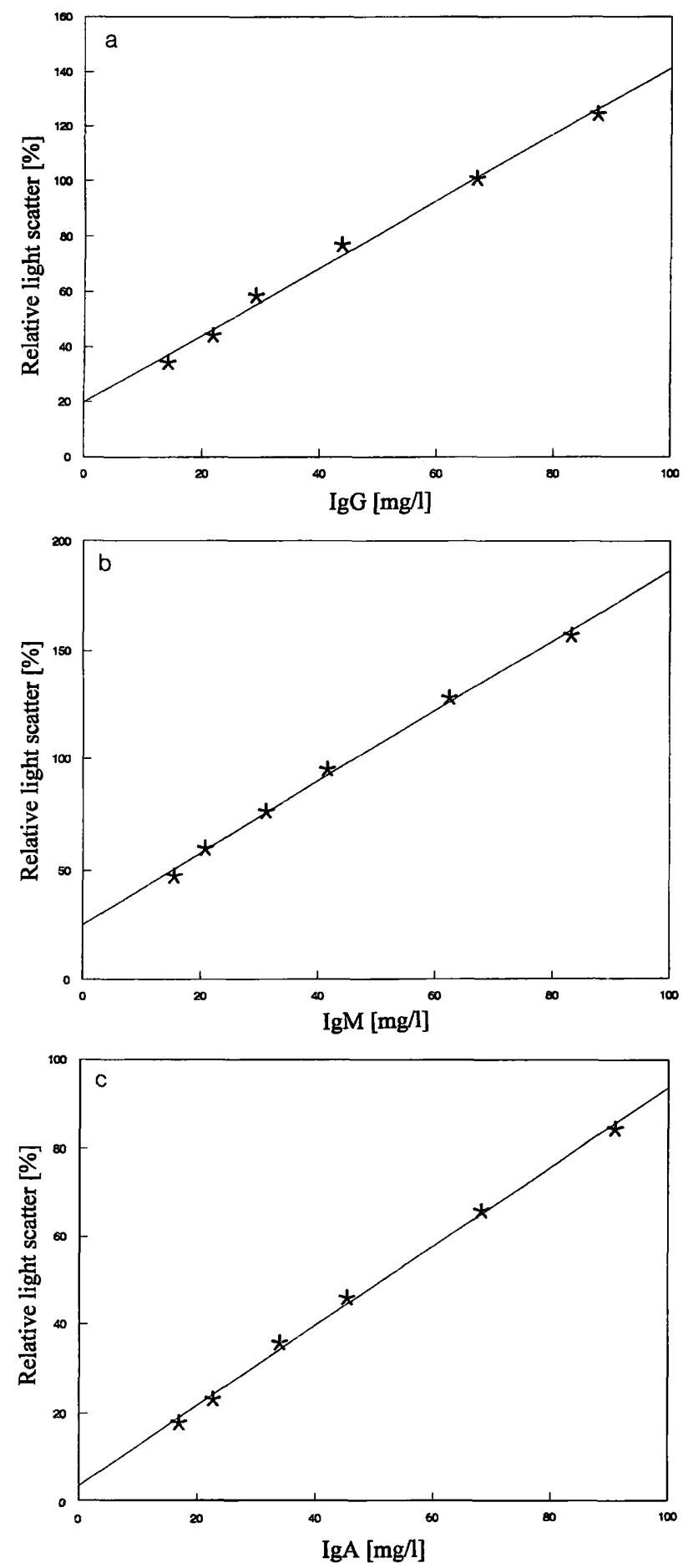

Fig. 1, a to c Laser nephelometry IgG, IgM and IgA standard reference curves for the determination of immunoglobulin concentrations in test samples. tained by each technique were compared by Spearman rank correlation analysis. Coefficients of correlation between laser nephelometry and single radial immunodiffusion were $r=0.9566$ for IgG, $r=0.9339$ for IgM, and $r=0.9082$ for IgA. Coefficients of correlation between laser nephelometry and ELISA were $r=0.9884$ for IgG, $r=0.9344$ for $\operatorname{IgM}$, and $r=0.9278$ for $\operatorname{IgA}$.

Mean immunoglobulin plasma concentrations were compared by one-way analysis of variance. Differences were not significant for mean IgG. Mean IgM concentrations determined by laser nephelometry were significantly higher than those determined by single radial immunodiffusion $(P=0.0102)$ and ELISA $(P=0.0102)$. Finally, the mean plasma IgA concentration determined by laser nephelometry was significantly lower $(P=0.0106)$ than that from the ELISA, whereas the differences between laser nephelometry and single radial immunodiffusion values were non-significant (tab. 2).

\section{Discussion}

Single radial immunodiffusion has traditionally been used for estimating immunoglobulin concentrations in body fluids. Unfortunately, its sensitivity is low, it is time consuming and requires 24 to 72 hours to obtain results, depending on the single radial immunodiffusion method followed. Hence, there is a need to replace it with an immunoassay lacking these drawbacks. The two-antibody-sandwich ELISA is highly sensitive and a useful method for detecting and measuring antigens (6, 9). The ELISA method can provide results the same day and allows a large number of samples to be processed in the same assay, but the method is time consuming and between-assay variation may be high if the variables are not carefully controlled. Also, its degree of automation is low making it less suitable for routine diagnostic immunoglobulin determination. Nephelometry of plasma immunoglobulins has several advantages over single

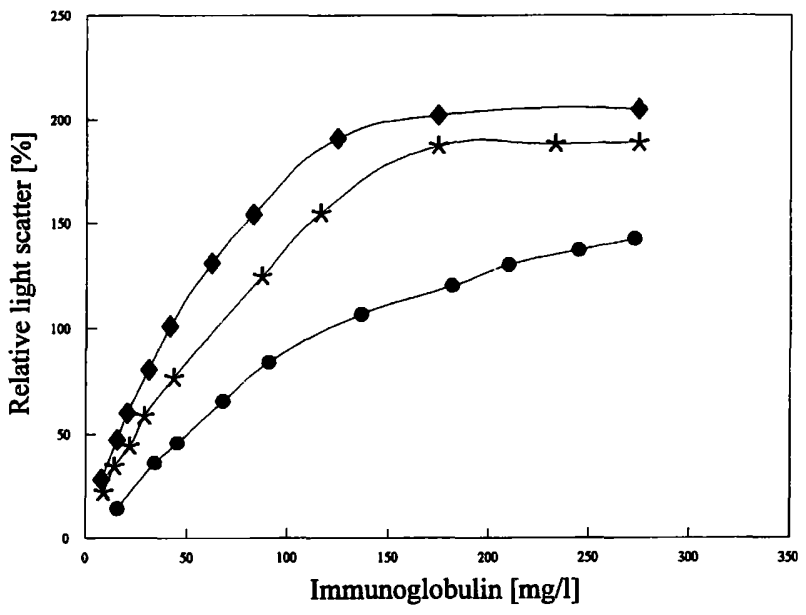

Fig. 2 Typical dilution curves obtained by laser nephelometry for the immunoglobulins assayed. 
Tab. 1 Between assay coefficient of variation (CV), sensitivity and analytical recovery of each immunoglobulin according to the method assayed.

\begin{tabular}{|c|c|c|c|c|c|c|c|c|c|}
\hline & \multicolumn{3}{|c|}{ CV (\%) } & \multicolumn{3}{|c|}{ Sensitivity $(\mathrm{g} / \mathrm{l})$} & \multicolumn{3}{|c|}{ Analytical recovery (\%) } \\
\hline & IgG & $\operatorname{IgM}$ & $\operatorname{IgA}$ & IgG & $\operatorname{IgM}$ & $\operatorname{Ig} \mathrm{A}$ & $\operatorname{lgG}$ & IgM & $\operatorname{IgA}$ \\
\hline Nephelometry & 4.12 & 6.98 & 6.35 & $1.9 \cdot 10^{-2}$ & $0.9 \cdot 10^{-2}$ & $1.7 \cdot 10^{-2}$ & 97.4 & 99.2 & 98.6 \\
\hline Radial immunodiffusion & 9.94 & 8.04 & 10.85 & 2.5 & 0.31 & 0.15 & 96.7 & 97.8 & 96.3 \\
\hline ELISA & 11.29 & 7.71 & 10.8 & $3.8 \cdot 10^{-5}$ & $2.0 \cdot 10^{-5}$ & $1.4 \cdot 10^{-5}$ & 97.3 & 98.1 & 95.4 \\
\hline
\end{tabular}

Tab. 2 Mean ( \pm SEM) plasma concentrations $(g / l)$ of IgG, IgM and IgA $(\mathrm{N}=44)$, and degree of significance between laser nephelometry and single radial immunodiffusion and ELISA.

\begin{tabular}{|c|c|c|c|c|c|}
\hline \multirow[t]{2}{*}{ Ig class } & \multirow{2}{*}{$\begin{array}{l}\text { Nephelometry } \\
\overline{\mathbf{x}} \pm \text { SEM }\end{array}$} & \multicolumn{2}{|c|}{ Radial immunodifusion } & \multicolumn{2}{|l|}{ ELISA } \\
\hline & & $\overline{\mathrm{x}} \pm \mathrm{SEM}$ & $\mathbf{P}$ & $\tilde{\mathrm{x}} \pm \mathrm{SEM}$ & $\mathbf{P}$ \\
\hline IgG & $16.377 \pm 1.178$ & $15.595 \pm 1.160$ & NS & $15.476 \pm 0.875$ & NS \\
\hline IgM & $1.829 \pm 0.077$ & $1.436 \pm 0.109$ & 0.01 & $1.474 \pm 0.108$ & 0.01 \\
\hline IgA & $0.210 \pm 0.008$ & $0.275 \pm 0.032$ & NS & $0.362 \pm 0.050$ & 0.01 \\
\hline
\end{tabular}

radial immunodiffusion and ELISA techniques. Nephelometers permit rapid analysis of many samples with one set of standards, thereby reducing the cost per analysis as compared with that of single radial immunodiffusion, and the method is less time-consuming that ELISA.

Recent years have seen an improvement in assay standardization and variability. However, examination of survey results between laboratories shows that the single radial immunodiffusion results exhibit great variability compared with nephelometry. In one specimen containing an approximately normal concentration of $\mathrm{IgG}$, the $\mathrm{CV}$ ranged from 3.9 per cent for laboratories using nephelometry to 41.2 per cent for laboratories using single radial immunodiffusion (1). In our study, the day-to-day precision of nephelometry was higher than that of the single radial immunodiffusion and ELISA methods. Previously reported values of between assay $\mathrm{CV}$ for the Mancini single radial immunodiffusion technique varied between 4 and 10 per cent, depending on the protein assayed, and about 7 to 12 per cent for the Fahey-McKelvey method (10). Similar CVs of about 10 per cent have been reported for ELISA assays (11).

For analysis of human samples, nephelometry replaced single radial immunodiffusion nearly two decades ago $(4,10)$. However, in nephelometric assays, non-turbid antisera are required to ensure low antibody blanks (3), and while these "nephelometry grade" antisera are commercially available for human immunoglobulin quantitation, to the authors' knowledge there are no equivalent antisera specific for canine immunoglobulins. However, whole plasma antisera can also be used if they are delipidated and turbidity is not excessive. Another potential problem in plasma immunoglobulin measurement is the formation of soluble immune complexes in the presence of antigen excess. Therefore samples whose antigen concentration greatly exceeds normal levels must be diluted and re-assayed within the range of the reference curve. Once appropriate dilutions of the standards and samples are prepared, quantitation can be completed in $60 \mathrm{~s}$ using kinetic measurements, or in 1 to $2 \mathrm{~h}$ using steady state or end-point analysis $(4,10)$. Mildly haemolysed samples do not generally interfere with immunoglobulin assays (1).

Although the ELISA method was more sensitive, one disadvantage was that it was only linear over approximately a $2-\log$ concentration range. Since the normal range of plasma immunoglobulins is wide, the ELISA procedures may require multiple dilutions of a patient's plasma (or repeated assays) to yield samples within the analytic range of the assay.

Statistical analysis of results by use of the KolmogorovSmirnov test showed a slightly skewed population distribution for the IgG and IgM distribution whereas the IgA concentrations were found to be non-Gaussian. These findings may be related to the already reported increase of plasma IgA with the age (15). Thus, in the sample studied, IgA concentrations tended to be lower in younger dogs (fig. 3), which together with the impossibility of IgA concentrations showing negative values can explain the non-Gaussian distribution observed. The correlation between nephelometric and single radial immunodiffusion values by use of Spearman rank coefficient was good for IgG and poorer for IgA. Previous studies comparing both techniques reported a slightly better correlation which varied from 0.98 to 0.94 depending on the immunoglobulin measured $(4,10)$.

Mean IgA concentration obtained by nephelometry was non-significantly lower than that obtained by single radial immunodiffusion, a finding which has been reported previously for human sera (4). However, in another 


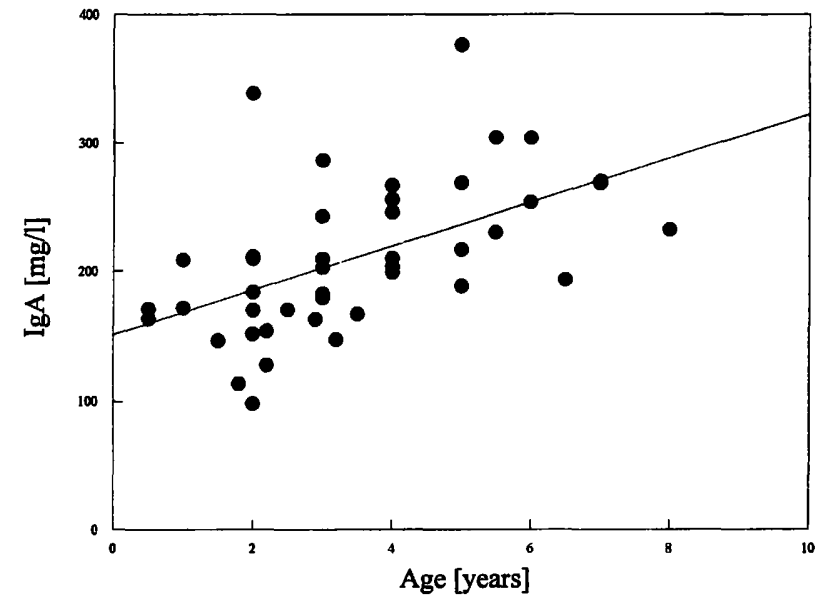

Fig. 3 Laser nephelometry distribution of IgA plasma concentrations as related to age $(\mathrm{N}=44)$.

study comparing nephelometry and single radial immunodiffusion for quantifying human plasma immunoglobulins, nephelometric results were consistently higher than single radial immunodiffusion results (10). The mean plasma IgA concentration determined by laser nephelometry was significantly lower than that determined by ELISA. In contrast, the nephelometric values were significantly higher for IgM than those obtained by single radial immunodiffusion and ELISA. Dilution curves of laser nephelometry for all immunoglobulins (fig. 2) showed appropriate slopes, and the linear part of each curve covered an optimal range of concentrations.

Two of the variables in the immunochemical quantitation of immunoglobulins are the antisera and the reference standard. Both reactants were the same in the three methods assayed, suggesting that other factors inherent to the techniques are responsible for the significant difference in the results obtained by these methods. Both laser nephelometry and single radial immunodiffusion depend on the ability of the antisera to produce precipitating immunocomplexes; thus one may reasonably expect closer quantitative results between these two techniques. However, the high precipitating capacity of IgM

\section{References}

1. Check IJ, Piper M, Papadea C. Immunoglobulin quantitation. In: Rose NR, De Macario EC, Fahey JL, Friedman H, Penn GM., editors. Manual of clinical laboratory immunology 4th ed. Washington: American Society of Microbiology, 1992: 71-83.

2. Willemse A, Noordzij A, Rutten VPG, Bernadina WE. Induction of non-IgE anaphylactic antibodies in dogs. Clin Exp Immunol 1985: 59:351-8.

3. Goudswaard J, Verdouw-Chamalaun CVM, Noordzij A. Quantitation of immunoglobulins in ovine sera and secretions by laser nephelometry. Comparison with the radial immunodiffusion (RID) technique. Vet Immunol Immunopathol 1980; $1: 163-77$

4. Deaton CD, Kameron WM, Smith RS, Creveling RL. Use of laser nephelometry in the measurement of serum proteins. Clin Chem 1976: 22;1465-71. complexes combined with the sensitivity of laser nephelometry, may be responsible for the higher IgM mean concentration obtained by this technique. Differences between IgA mean concentrations as determined by nephelometry and ELISA were quantitatively more important. The ELISA method detects the amount of bound antibody, and since its sensitivity is much higher the contribution of minor unspecific reactions to the amount of colour measured may be more important.

As determined by nephelometry, the plasma concentrations of immunoglobulins IgG and IgM are in the same range as those already reported, which were determined mostly by single radial immunodiffusion (12), whereas the mean plasma IgA concentration was slightly below some of the previously reported values for the dog $(0.26$ to $1.24 \mathrm{~g} / 1)(12,13)$. Widely varying normal ranges of canine IgA plasma immunoglobulins have been reported by different authors, and the normal range has been shown to vary, inter alia, with the age and breed (14, 15). Thus, reference values for each laboratory will vary depending on the individuals used to establish them. As the single radial immunodiffusion mean IgA value $(0.275 \mathrm{~g} / 1)$ was not significantly different, and the ELISA mean value is also in the lower range, it can be assumed that this low IgA concentration was mostly attributable to the sample analysed and not to the technique.

\section{Conclusion}

Laser nephelometry is a reproducible and accurate technique for quantitating immunoglobulins in canine plasma. Results are produced in a few hours and correlation with other available methods is acceptable. Although without clinical relevance, the presence of some statistically significant differences between laser nephelometry and the other analytical methods assayed indicate that a specific reference interval for immunoglobulin concentrations determined by nephelometry should be established by each laboratory.

5. Mancini G, Carbonara AO, Heremans JF. Immunochemical quantitation of antigens by single radial immunodiffusion. Immunochemistry 1965: 2:235-54.

6. Ginel PJ, Margarito JM, Molleda JM, López R, Novales M, Bernadina W. Biotin-avidin amplified enzyme-linked immunosorbent assay (ELISA) for the measurement of canine serum IgA, IgG, and IgM. Res Vet Sci 1996; 60:107-10.

7. Schwartz D. Métodos estadisticos para médicos y biologos. Barcelona: Herder SA, 1985:251-313.

8. Büttner J, Borth J, Boutwell R, Broughton PMG, Bowyer RC. International Federation of Clinical Chemistry. Part 2. Assessment of analytical methods for routine use. J Clin Chem Clin Biochem 1980; 18:78-88.

9. Harlow E, Lane D, editors. Immunoassays. In: Antibodies: a laboratory manual. New York: Cold Spring Harbor Laboratory, 1988: $553-612$. 
10. Alexander RL. Comparison of radial immunodiffussion and laser nephelometry for quantitating some serum proteins. Clin Chem 1980; 26:314-7.

11. Papadea D, Check IJ, Reimer CB. Monoclonal antibody-based solid-phase immunoenzymometric assays for quantifying human immunoglobulin $G$ and its subclasses in serum. Clin Chem 1985; 31:1940-5.

12. Gorman NT, Halliwell RWE. Immunoglobulin quantitation and clinical interpretation. In: Halliwell REW, Gorman NT, editors. Veterinary clinical immunology. Philadelphia: Saunders, 1989:55-73.

13. Day MJ, Penhale WJ. Serum immunoglobulin A concentrations in normal and diseased dogs. Res Vet Sci 1988; 45:360-3.
14. Reynolds HY, Johnson JS. Quantitation of canine immunoglobulins. J Immunol 1970; 105:698-703.

15. Schreiber M, Kantimm D, Kirchhoff D, Heimann G, Bhargava AS. Concentrations of IgG, IgM and IgA and their age-dependence in beagle dogs as determined by a newly developed enzyme-linked-immunosorbent-assay (ELISA). Eur J Clin Chem Clin Biochem 1992; 30:775-8.

Received August 26/December 6, 1996

Corresponding author: Dr. Pedro J. Ginel, Departmento de Patología, Clínica Veterinaria, Facultad de Veterinaria, Avda. Medina Azahara 9, E-14005 Córdoba, Spain 